\title{
Clinical Characteristics of 18-Trisomy Syndrome Failed to Be Diagnosed After Prenatal Screening
}

\author{
Yishan Dong* \\ Changzhou Maternity and Child Health Care Hospital affiliated to Nanjing Medical University, China
}

*Corresponding author: Yishan Dong, Changzhou Maternity and Child Health

Care Hospital affiliated to Nanjing Medical University, China.

\section{Abstract}

Objective: To discover the characteristics of trisomies 18 who were prenatal diagnosed or failed to be diagnosed, and to reduce the failed to be diagnosed.

Method: In this multicenter study, we collected a total of 52 babies of 18-trisomy syndrome (34 cases were prenatally diagnosed and 18 failed to be diagnosed) and analyzed their characteristics.

Results: In the past 13 years, according to the data from the prenatal diagnosis center in Jiangsu, the estimated detection rate was $65.4 \%$ for trisomies 18 in the second trimester. After analyzed the distribution of the groups according to T18-risk, there were two important cut-off values to be noted: $1 / 455$ and 1/5050. It was worthwhile noting that the risk of $65.4 \%$ trisomy 18 cases was higher than $1 / 455,90.4 \%$ higher than $1 / 5050$. However, $96.1 \%$ cases in normal control group were lower than $1 / 5050$. No case from normal control group would be higher than the value of risk in 1/450. Meanwhile, the T18-risk of $50 \%$ T18 failed to be diagnosed cases were 1/1001 1/5050. If we chose NIPT follow the standard of $1 / 5050$, we could find $90.4 \%$ T18 cases. It could increase $19.2 \%$ T18 babies be detected, but 3.2\% normal mother would accept the NIPT examination which maybe unnecessary.

Conclusions: The prediction mode of HCG MoM and AFP MoM might be able to help us reduce the failed to be diagnosed. It is also necessary to adjust more reasonable range of NIPT with further clinical researches.

Keywords: 18 trisomy syndrome; Prenatal screening; Biomarkers; Prenatal diagnosis; Non-invasive prenatal testing

What Is Already Known on This Subject?

It is well known that prenatal screening and diagnosis is the effective way to avoid the birth of trisomy 18. However, there are still some cases will be missed.

What Do the Results of This Study Add?

There were two important cut-off value of prenatal screening to be noted: $1 / 455$ and 1/5050. The T18-risk of $50 \%$ T18 failed to be diagnosed cases were $1 / 1001 \sim 1 / 5050$. If we chose NIPT follow the standard of $1 / 5050$, we can find $90.4 \%$ T18 cases. It can increase $19.2 \%$ T18 babies be detected, while add $3.2 \%$ normal mother accepted the NIPT.

What are the Implications of These Study?

The prediction mode of HCG MoM and AFP MoM might be able to help us reduce the failed to be diagnosed. It is also necessary to adjust more reasonable range of NIPT with further clinical researches. 


\section{Introduction}

Edwards syndrome, also named as 18-trisomy syndrome, is the common chromosomal abnormalities with an incidence of 1 in 3000 8000 in live births [1]. Due to an extra chromosome 18, the main clinical manifestations are multiple malformations and severe mental retardation, which caused only few children could survive the first year and very few cases could live longer [1,2]. According clinical researches, about $50 \%$ of babies with trisomy 18 could live longer than one week and about $5-10 \%$ of children beyond the first year [3]. So, it seriously affected the health of children. It is well known that the program of prenatal screening and diagnosis is the effective way to avoid the birth of trisomy 18 baby.

In the past three decades, prenatal screening for 18-trisomy syndrome has been widely used in pregnant women worldwide $[4,5]$ which based on screening by maternal age, maternal serum markers screening, or detection of sonographic abnormalities (e.g., increased nuchal translucency thickness, growth retardation, choroid plexus cyst, overlapping of fingers, and congenital heart defects) [3]. The most common prenatal screening method for 18-trisomy syndrome is the serological prenatal screening in the second trimester, which detect the serum levels of free beta subunit of human chorionic gonadotropin ( $\mathrm{f} \beta \mathrm{hCG}$ ) and alpha-fetoprotein (AFP) with maternal age [6,7]. The approximately detection rate was $57 \%$ with $0.7 \%$ false positive rate (Spencer K 1999). Effective screening can be carrying out in the first trimester screening (between 8 and 13 weeks), combination of serum levels of $\mathrm{f} \beta \mathrm{hCG}$, pregnancy-associated plasma protein A (PAPP-A), maternal age and ultrasound nuchal translucency (NT) measurements. The estimated detection rate was $87.0 \%$ for Down syndrome and $91.8 \%$ for 18-trisomy syndrome and trisomies 13 with the false-positive rate of $2.2 \%$ [9]. So, regardless of the kind of prenatal screening, it still had some cases of 18-trisomy syndrome would miss diagnosis because of the technical limitations of the prenatal screening.

Table 1: The baseline characteristics of three groups in this study.

\begin{tabular}{|c|c|c|c|c|}
\hline & T18 prenatal diagnosed group & T18 miss diagnosed group & Normal control group & P-value \\
\hline N & 34 & 18 & 309 & 309 \\
\hline Ethnicity (Chinese) & 34 & 18 & 309 & $28.00 \pm 3.71$ \\
\hline Singleton pregnancy & 34 & 18 & $58.88 \pm 10.47$ & 0.93 \\
\hline Maternal Age (years) & $30.07 \pm 5.71$ & $28.73 \pm 3.34$ & $16.88 \pm 0.95$ & 0.792 \\
\hline Maternal Weight (kg) & $58.51 \pm 9.29$ & $58.04 \pm 7.17$ & $16.85 \pm 1.35$ & \\
\hline Gestational age (weeks) & $16.72 \pm 1.02$ & & & \\
\hline
\end{tabular}

\section{Methods}

Samples Collect: After prenatal genetic counseling and informed consent, the women received the prenatal screening in second trimester. According the operating program of prenatal screen, we collected the blood from every case in 2nd trimester $(15 w \sim 20 w)$. Gestational age was calculated by each pregnant woman's last menstrual period or ultrasonography. $3 \mathrm{ml}$ blood were collected by simple needle aspiration and centrifuged at $3000 \mathrm{rpm}$ for 5 minutes after being placed $0.5 \mathrm{~h}$ at room temperature. The
Recently, non-invasive prenatal testing (NIPT), detection of fetal cell-free DNA (cfDNA) in maternal plasma, was proved to be a better prenatal screening program for common fetal aneuploidies. Nowadays, NIPT was widely used to prenatal screen the T21, T18, T13 [10]. Some societies and organizations, such as ACOG [11] and ISPD [12], have issued the committee opinions or guidelines about the clinical application of NIPT, and they both recommend the patients of high-risk group to accept NIPT. In 2015, Chinese scholars firstly suggested the mothers whose T18 screening results were intermediate risk $(1 / 350 \sim 1 / 1000)$ to accept NIPT to reduce the failed to be diagnosed.

However, it is necessary to adjust more reasonable range of NIPT with further clinical researches.

In the present study, we have collected the cases of trisomies 18 who were prenatal diagnosed or failed to be diagnosed from the prenatal diagnosis center and analyzed their characteristics. With statistical analysis, we hope to be able to improve the programs of prenatal screening and reduce the failed to be diagnosed of 18-trisomy syndrome.

\section{Materials and Methods}

\section{Patients and Design}

The study design and protocol were reviewed and approved by the ethics committee of Changzhou Maternity and Child Health Care Hospital affiliated to Nanjing Medical University. From October 2002 to June 2015 , the pregnant women received the prenatal screening in the hospital. A total of 52 babies of 18-trisomy syndrome were found in the center, included 34 cases were prenatally diagnosed and 18 cases failed to be diagnosed. We selected the objects of their mothers. Meanwhile, 309 mothers were selected as the normal control group. They accepted prenatal screening in our hospital and were confirmed have normal babies after followed-up. Both common characteristics were shown in Table 1.

samples were stored at $4{ }^{\circ} \mathrm{C}$ within 7 days and $-80{ }^{\circ} \mathrm{C}$ for long-stem.

Prenatal screening in second trimester: As Miao ZY, et al. [13] described, the levels of AFP and $\mathrm{f} \beta \mathrm{hCG}$ were quantified by time-resolved fluoroimmunoassay (TRFIA) using Wallac 1235 AutoDELFIA (DELFIA1235: Perkin Elmer, Waltham, MA) in the center. The value was also presented as multiples of the median [14] and determined the risk of carrying a fetus with 18-trisomy syndrome with Wallace LifeCycleTM Elipse analysis software (Perkin Elmer) in the second trimester. 
Statistical analysis: With EmpowerStats x64 software [15], the statistical analysis was performed, including stratified analysis, receiver operating characteristic curve (ROC curve), interaction test, covariate screening and curve fitting. T test and non-parametric test were used to compare the differences for continuous variables between two groups. $\mathrm{P}<0.05$ is chosen to be statistically significant.

\section{Results}

In the past 13 years, we found a total of 52 women carried 18-trisomy syndrome baby. Among 52 cases, 34 were prenatally diagnosed by amniocentesis or pencutaneous umbilical blood sampling after serum screening. However, a total of 18 babies were

Table 2: Compared the value of $\mathrm{f} \beta \mathrm{hCG}$ and AFP between the groups.

\begin{tabular}{|c|c|c|c|c|c|}
\hline & $\mathbf{n}$ & $\mathbf{f} \beta \mathbf{h C G}(\mathbf{n g} / \mathbf{m l})$ & HCG MoM & AFP (U/ml) & AFP MoM \\
\hline T18 groups & 52 & & & & \\
\hline T18 prenatal diagnosed group & 34 & $2.09(1.80-2.62)$ & $0.17(0.12-0.21)$ & $22.09 \pm 9.49$ & $0.59 \pm 0.25$ \\
\hline T18 miss diagnosed group & 18 & $5.46(4.91-6.56)$ & $0.43(0.33-0.52)$ & $20.75 \pm 6.94$ & $0.55 \pm 0.20$ \\
\hline Normal control group & 309 & $13.90(9.36-21.88)$ & $0.91(0.63-1.42)$ & $42.97 \pm 15.15$ & $1.10 \pm 0.36$ \\
\hline P-value & & $<0.001$ & $<0.001$ & $<0.001$ & $<0.001$ \\
\hline
\end{tabular}

\section{Note:}

The normal distribution data were expressed as mean \pm SD .

The abnormal distribution data were expressed as median (P2.5-P97.5).

Table 3: Association of $\mathrm{f} \beta \mathrm{hCG}$ and AFP levels with T18 events.

\begin{tabular}{|c|c|c|c|c|}
\hline & Total & Odds ratios* & 95\%CI & P value \\
\hline $\mathrm{f} \beta \mathrm{hCG}(\mathrm{ng} / \mathrm{ml})$ & $12.55(7.65-20.01)$ & 0.68 & $(0.61,0.76)$ & $<0.0001$ \\
\hline HCG MoM & $0.84(0.52-1.36)$ & 0 & $(0.00,0.02)$ & $<0.0001$ \\
\hline AFP $(\mathrm{U} / \mathrm{ml})$ & $40.10 \pm 16.18$ & 0.79 & $(0.74,0.84)$ & $<0.0001$ \\
\hline AFP MoM & $1.02 \pm 0.39$ & 0 & $(0.00,0.00)$ & $<0.0001$ \\
\hline
\end{tabular}

Note: *Adjust for: AGE; WEIGHT, Gestational age. Odds ratios and 95\% confidence intervals are presented to show the risk of T18 syndrome.

After analyzed the distribution of the groups according to T18risk, there were two important cut-off values worthy to be noted: $1 / 455$ and 1/5050. After ROC analysis, 1/5050 was considered as the best optimal cut-off value with the sensitivity, specificity, positive predictive value (PPV) and negative predictive value (NPV) were $90.4 \%, 96.1 \%, 79.7 \%$ and $98.3 \%$ respectively. While the PPV and accuracy of the cut-off in $1 / 455$ were $100 \%$ and $99.1 \%$ respectively. So, we compared the distribution of the groups by $1 / 455$ and $1 / 5050$, as saw as Table 4 . It was worthwhile noting that the risk of $65.4 \%$ trisomy 18 cases was higher than $1 / 455,90.4 \%$ higher than $1 / 5050$. However, $96.1 \%$ cases in normal control group still failed to be diagnosed in the prenatal diagnosis center, although their mothers also received the prenatal screening. However, we didn't discover their abnormal risk. The estimated detection rate was $65.4 \%(34 / 52)$ for the prenatal screening and diagnosis program of trisomies 18 in the second trimester.

Compared with normal control group, all of the level of $\mathrm{f} \beta \mathrm{hCG}$, HCG MoM, AFP and AFP MoM were significant decreased (Table 2) in T18 groups. As shown as Table 3, the Odds ratios (OR) and 95\% confidence intervals were established after the regression analyzed with adjusting the maternal age and weight. Notedly, both of the HCG-MoM and AFP-MoM were the important influential factors of 18-trisomy syndrome $(\mathrm{OR}=0.00)$.

Table 4: Compared the distribution of the groups according to T18-risk, $n(\%)$.

\begin{tabular}{|c|c|c|c|c|}
\hline T18-risk & T18 groups & T18 prenatal diagnosed group & T18 miss diagnosed group & Normal control group \\
\hline$>1 / 455$ & $34(65.4)$ & $31(91.2)$ & $3(16.7)$ & 0 \\
\hline $1 / 455 \sim 1 / 1000$ & $3(5.8)$ & 0 & $3(16.7)$ & $2(0.6)$ \\
\hline $1 / 1001-1 / 5050$ & $10(19.2)$ & $1(2.9)$ & $9(50.0)$ & $10(3.2)$ \\
\hline$<1 / 5050$ & $5(9.6)$ & $2(5.9)$ & $3(16.7)$ & $297(96.1)$ \\
\hline Total & $52(100)$ & $34(100)$ & $18(100)$ & $309(100)$ \\
\hline
\end{tabular}




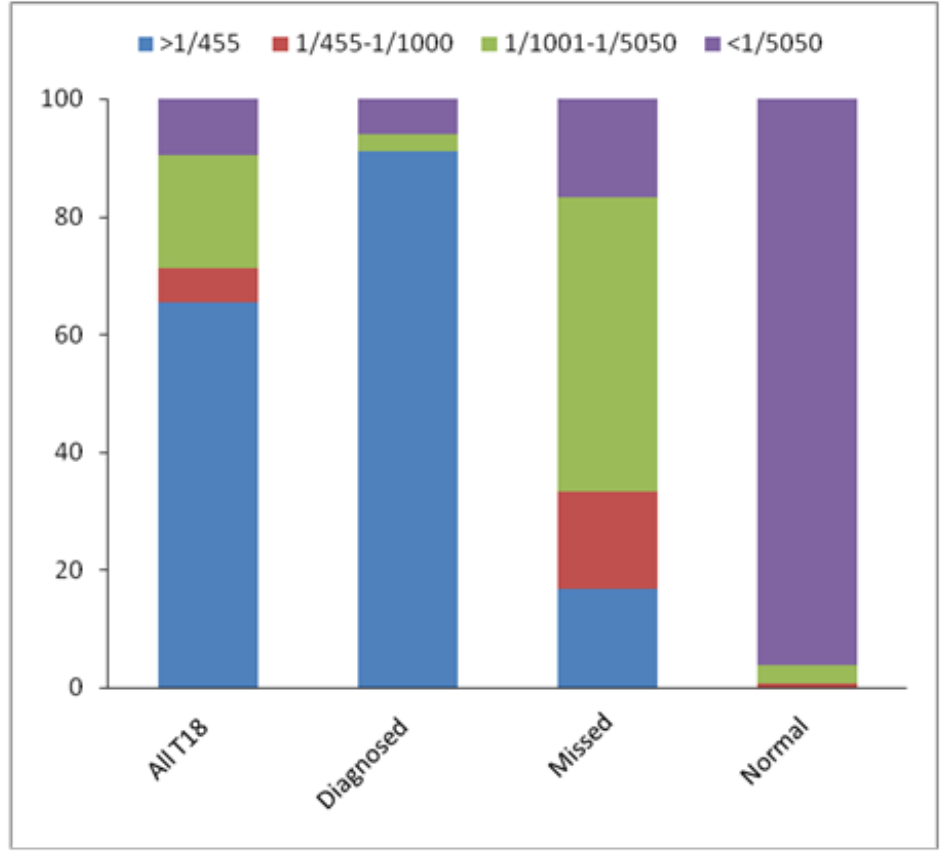

Figure 1: The distribution of T18-RISK in different group

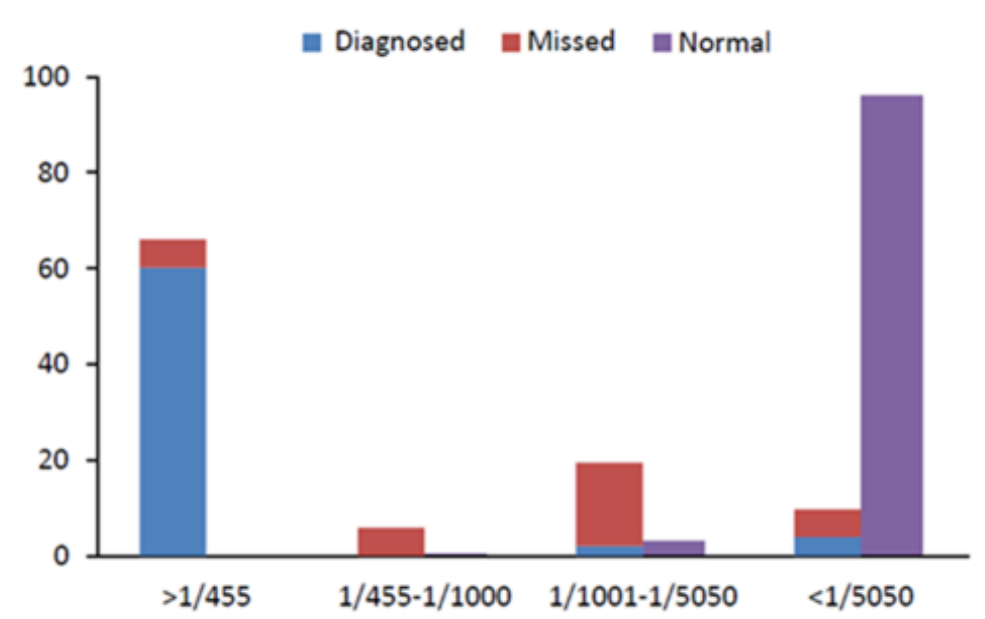

Figure 2: The distribution of three groups according to T18-RISK.

\section{Discussion}

It is well known that prenatal screening can contribute to avoidance the birth of 18-trisomy syndrome. However, it still needs improving greatly. In present study, we collected the 18-trisomy syndrome whose mothers received the prenatal screening program from the centers. After analyzed their characteristics and statistical analysis, it maybe helps to improve the effectiveness of prenatal screening, especially the choice of NIPT.

At first, we found that all of the level of f 3 CG, HCG MoM, AFP and AFP MoM were significant decreased in 18-trisomy syndrome group. In the common prenatal screening program, we calculated the T18-risk combined with maternal age, gestational week, the concentration of $\mathrm{f} \beta \mathrm{hCG}$ and AFP. We have already considered the effectuation of the value HCG MoM and AFP MoM to T18-risk.
However, maybe we could make use of them better to improve prenatal screening program.

Second, it was worthwhile noting that the characteristic of the distribution according to T18-risk. It is well known that the value of risk is the most important index in prenatal screening program, and it also used to accept NIPT or not. ACOG(2015) and ISPD(Benn $P$ 2015) recommend the patients of high-risk group to accept NIPT. Chinese scholars firstly suggested the mothers whose DS screening results were intermediate risk $(1 / 300 \sim 1 / 1000)$ to accept NIPT to reduce the failed to be diagnosed. According the results of present study, we recommended to pay attention to the two cut-offs: $1 / 455$ and $1 / 5050$. It was very interesting that the positive predictive value and the accuracy of $1 / 455$ was $100 \%$ and $99.1 \%$ respectively. $65.4 \%$ trisomy 18 cases were higher than $1 / 455$, while no normal 
pregnant women would be higher than $1 / 450$. It meant that if the T18-risk of a pregnant woman was higher than $1 / 455$, the risk of her fetal was 18-trisomy syndrome almost close to $100 \%$. So, the women should be recommended to directly accept the cytology of prenatal diagnosis, such as amniocentesis or pencutaneous umbilical blood sampling. Meanwhile, the T18-risk of $96.1 \%$ normal pregnant woman were lower than $1 / 5050$, only $9.6 \%$ in T18 group. So, we suggested the women whose T18-risk $<1 / 5050$ could accept the ultrasonic check in the future of pregnancy. What could we do with the women in $1 / 455$ to $1 / 5050$ ? The present point is the women who's the T18-risk were higher than $1 / 1000$ should accept NIPT. However, we found $71.2 \%$ T18 cases could be diagnosed according the standard, while $38.8 \%$ might still missed. The T18-risk of 50\% T18 failed to be diagnosed cases were in the section of $1 / 1001 \sim 1 / 5050$. We preliminary recommended adjust the standard to $1 / 5050$, and we could find $90.4 \%$ women carries T18 cases by NIPT. It could increase $19.2 \%$ T18 babies be detected, but 3.2\% normal mother would receive unnecessary check.

In conclusion, we collected the 18 trisomy syndrome cases from the prenatal diagnosis center and analyzed their characteristics to help us reduce the failed to be diagnosed. It is also necessary to adjust more reasonable range of NIPT with further clinical researches.

\section{Acknowledgment}

We thank all the participants for their contribution to this study

\section{Details of Ethics Approval}

The study design and protocol were reviewed and approved by the ethics committee of Changzhou Maternity and Child Health Care Hospital affiliated to Nanjing Medical University (No. 2017003). All pregnant women received genetic counseling and provided informed consent before testing. All procedures performed in studies involving human participants were in accordance with the ethical standards of the institutional or national research committee, and with the 1964 Helsinki Declaration and its later amendments or comparable ethical standards.

\section{Conflict of Interest}

Author declare that they have no competing interests.

\section{References}

1. Lin HY, Lin SP, Chen YJ, Hung HY, Kao HA, , et al. (2006) Clinical characteristics and survival of trisomy 18 in a medical center in Taipei, 1988-2004. Am J Med Genet A 140(9): 945-951.

2. Niedrist D, Riegel M, Achermann J, Schinzel A (2006) Survival with trisomy 18--data from Switzerland. Am J Med Genet A 140(9): 952-959.

3. Cereda A, Carey JC (2012) The trisomy 18 syndrome. Orphanet J Rare Dis 7: 81.

4. Wald NJ (2010) Prenatal screening for open neural tube defects and Down syndrome: three decades of progress. Prenat Diagn 30(7): 619621.

5. Maxwell S, Bower C, O'leary P (2015) Impact of prenatal screening and diagnostic testing on trends in Down syndrome births and terminations in Western Australia 1980 to 2013. Prenat Diagn 35(13): 1324-1330.

6. Cuckle HS, Wald NJ, Thompson SG (1987) Estimating a woman's risk of having a pregnancy associated with Down's syndrome using her age and serum alphafetoprotein level BJOG 94(5): 387-402.

7. Wald NJ, Cuckle HS, Densem JW, Nanchahal K, Royston P, et al. (1988) Maternal serum screening for Down's syndrome in early pregnancy. BMJ 297(6653): 883-887

8. Spencer K (1999) Second trimester prenatal screening for Down's syndrome using alpha-fetoprotein and free beta hCG: a seven year review. BJOG 106(12): 1287-1293.

9. Wright D, Syngelaki A, Bradbury I, Akolekar R, Nicolaides KH (2014) First-trimester screening for trisomies 21,18 and 13 by ultrasound and biochemical testing. Fetal Diagn Ther 35(2): 118-126.

10. Gil MM, Quezada MS, Revello R, Akolekar R, Nicolaides KH (2015) Analysis of cell-free DNA in maternal blood in screening for fetal aneuploidies: updated meta-analysis. Ultrasound Obstet Gynecol 45(3): 249-266.

11. (2015) Committee Opinion No. 640: Cell-free DNA Screening for Fetal Aneuploidy. Obstet Gynecol 126(3): e31-e37.

12. Benn P, Borrell A, Chiu RWK, Cuckle H, Dugoff L, et al. (2015) Position statement from the Chromosome Abnormality Screening Committee on behalf of the Board of the International Society for Prenatal Diagnosis. Prenat Diagn 35(8): 725-734.

13. Miao ZY, Liu X, Shi TK, Xu Y, Song QH, et al. (2012) First trimester, second trimester, and integrated screening for Down's syndrome in China. J Med Screen 19(2): 68-71.

14. Esplin MS, Elovitz MA, Iams JD, Parker CB, Wapner RJ, et al. (2017) Predictive Accuracy of Serial Transvaginal Cervical Lengths and Quantitative Vaginal Fetal Fibronectin Levels for Spontaneous Preterm Birth Among Nulliparous Women. JAMA 317(10): 1047-1056.

15. Wu J, Geng J, Liu L, Teng W, Liu L, et al. (2015) The Relationship between Estimated Glomerular Filtration Rate and Diabetic Retinopathy. J Ophthalmol 2015: 326209 